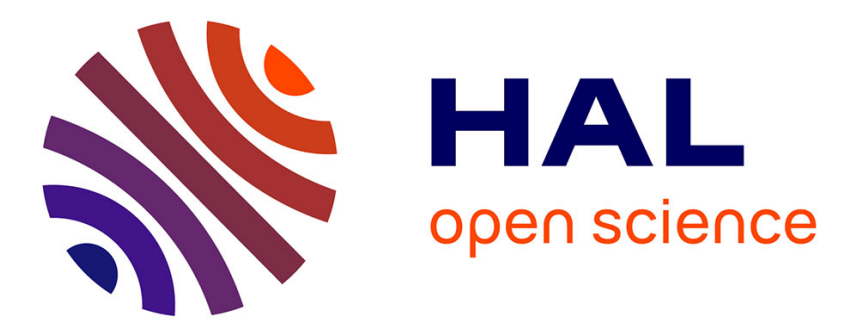

\title{
Optical linewidth of a passively mode-locked semiconductor laser
}

Tatiana Habruseva, Shane O’Donoghue, Natalia Rebrova, Fabien Kéfélian, Steve Hegarty, Guillaume Huyet

\section{- To cite this version:}

Tatiana Habruseva, Shane O'Donoghue, Natalia Rebrova, Fabien Kéfélian, Steve Hegarty, et al.. Optical linewidth of a passively mode-locked semiconductor laser. Optics Letters, 2009, 34, pp.33073309. 10.1364/OL.34.003307 . hal-01075182

\section{HAL Id: hal-01075182 \\ https://hal.science/hal-01075182}

Submitted on 16 Oct 2014

HAL is a multi-disciplinary open access archive for the deposit and dissemination of scientific research documents, whether they are published or not. The documents may come from teaching and research institutions in France or abroad, or from public or private research centers.
L'archive ouverte pluridisciplinaire HAL, est destinée au dépôt et à la diffusion de documents scientifiques de niveau recherche, publiés ou non, émanant des établissements d'enseignement et de recherche français ou étrangers, des laboratoires publics ou privés. 


\title{
Optical linewidth of a passively mode-locked semiconductor laser
}

\author{
T. Habruseva, ${ }^{1,2, *}$ S. O’Donoghue, ${ }^{1,2}$ N. Rebrova, ${ }^{1,2}$ F. Kéfélian,,${ }^{1,2,3}$ S. P. Hegarty, ${ }^{1,2}$ and G. Huyet ${ }^{1,2}$ \\ ${ }^{1}$ Tyndall National Institute, Lee Maltings, Cork, Ireland \\ ${ }^{2}$ Department of Applied Physics and Instrumentation, Cork Institute of Technology, Cork, Ireland \\ ${ }^{3}$ Present address: Laboratoire de Physique des Lasers, Université Paris 13 and CNRS, \\ 99 Avenue Jean-Baptiste Clement, 93430 Villetaneuse, France \\ *Corresponding author: tatiana.gabruseva@gmail.com
}

Received July 6, 2009; revised September 18, 2009; accepted September 27, 2009; posted October 2, 2009 (Doc. ID 113905); published October 23, 2009

\begin{abstract}
We measured the optical linewidths of a passively mode-locked quantum dot laser and show that, in agreement with theoretical predictions, the modal linewidth exhibits a parabolic dependence with the mode optical frequency. The minimum linewidth follows a Schawlow-Townes behavior with a rebroadening at high power. In addition, the slope of the parabola is proportional to the RF linewidth of the laser and can therefore provide a direct measurement of the timing jitter. Such a measurement could be easily applied to modelocked semiconductor lasers with a fast repetition rate where the RF linewidth cannot be directly measured. (C) 2009 Optical Society of America

OCIS codes: $140.4050,230.5590$.
\end{abstract}

Optical frequency combs have become important tools for time and frequency metrology [1]. They have also been identified as potential sources for coherent communications and signal processing [2]. Optical frequency combs are commonly generated by modelocked lasers (MLLs) that periodically emit short pulses with an optical spectrum composed of a set of equally spaced narrow lines. Some metrology applications require octave spanning combs generated by Ti:sapphire lasers where the pump intensity and the cavity length fluctuations constitute the main sources of noise. Coherent communications and signal processing require a much narrower frequency line set that can be generated by monolithic passively mode-locked semiconductor lasers where the spontaneous emission constitutes the main source of noise in a large Fourier frequency range. In recent years monolithic quantum dot (QD) MLLs have shown much promise in producing stable pulses, of the order of picoseconds, with high repetition rates and low timing jitter [3-5].

In general, noise influences mainly the amplitude, the central optical frequency, the comb line to comb line frequency spacing, the pulse-to-pulse timing, and the optical phase of mode-locked semiconductor lasers, but the broadening of the comb lines [6] is dominated by the contributions of optical phase noise and pulse-to-pulse timing fluctuations. The quantum limited fluctuations of the optical phase induce a Lorentzian line shape in all the comb lines, similarly to the Schawlow-Townes line shape of single-mode lasers. The optical linewidth is also related to timing jitter fluctuations. The stochastic dynamics of MLLs can be described by a set of Langevin equations [7] capturing the evolution of the power, frequency, timing jitter, and optical phase of the laser. By retaining only the timing jitter and the optical phase fluctuations, the optical field is [8]

$$
A(t)=\sum_{m=-\infty}^{\infty} a\left(t-m T_{r}-\Delta t(m)\right) e^{-i \omega_{0}\left(t-m T_{r}\right)-i \phi},
$$

where $a(t)$ is the optical pulse envelope, $\phi=\Delta \theta(m)$ $+\Phi_{\mathrm{CE}}(m), T_{r}$ is the device repetition rate, $\omega_{0}$ is the center frequency, and $\Phi_{\mathrm{CE}}(m)$ is the carrier-envelope phase shift of the $m$ th pulse. The functions $\Delta t(m)$ and $\Delta \theta(m)$ are random variables that describe the influence of noise on the pulse-to-pulse timing and optical phase. Close to the lines, the functions $\Delta t(T)$ and $\Delta \theta(T)$ can be approximated as Gaussian random walk processes with $\left\langle(\Delta \theta(T)-\Delta \theta(0))^{2}\right\rangle=2 \Delta \omega_{0}|T|$ and $\left\langle(\Delta t(T)-\Delta t(0))^{2}\right\rangle=2 \Delta \omega_{\mathrm{RF}}|T|\left(T_{r} / 2 \pi\right)^{2}$. Following [8], a set of Lorentzian lines with frequencies $\omega_{n}$ and linewidths $\Delta \omega_{n}$ compose the optical spectrum

$$
S(\omega) \sim\left|\hat{a}\left(\omega-\omega_{0}\right)\right|^{2} \sum_{n=-\infty}^{\infty} \frac{2 \Delta \omega_{n}}{\left(\omega-\omega_{n}\right)^{2}+\Delta \omega_{n}^{2}},
$$

where $\hat{a}$ is the Fourier transform of the pulse envelope. The power spectrum of the photocurrent RF signal is a set of Lorentzians centered at $m \omega_{r}=2 m \pi / T_{r}$ with the linewidth of $m$ th harmonic denoted by $\Delta \omega_{\mathrm{RF}, m}$,

$$
S_{\mathrm{RF}}(\omega) \sim \sum_{m=-\infty}^{\infty} \frac{2 \Delta \omega_{\mathrm{RF}, m}}{\left(\omega-m \omega_{r}\right)^{2}+\Delta \omega_{\mathrm{RF}, m}^{2}} .
$$

The values $\Delta \omega_{n}$ and $\Delta \omega_{\mathrm{RF}, 1}$ are related via

$$
\Delta \omega_{n}=\Delta \omega_{0}+\Delta \omega_{\mathrm{RF}, 1}\left(n-n_{0}\right)^{2},
$$

where $n$ is the mode number and $n_{0}$ is the mode number corresponding to the minimum linewidth.

Equation (4) shows that the parabolic increase in the optical linewidth with the mode number is proportional to the first harmonic of the RF linewidth and is therefore directly related to the timing jitter. Although such a behavior was predicted in [8] and a parabolic dependence was measured in [9], there is - to our knowledge-no detailed experimental investigation of the relationship between the modal optical linewidth and the timing jitter in passively MLLs. 
In this Letter, we measure the optical linewidth of the modes of a mode-locked QD laser and demonstrate that the parabolic increase can be directly related to the RF linewidth. As a result, the timing jitter of a device can be evaluated from a measurement of the optical linewidth of the various modes of the laser. In addition, we show that the minimum optical linewidth follows a Schawlow-Townes-like dependence but with a rebroadening at high currents, similar to that observed in single mode QD lasers [10].

The devices used in this study were two-section monolithic InAs/GaAs QD ridge waveguide lasers emitting at $1.3 \mu \mathrm{m}$, similar to those used in [11]. The devices were cleaved at a length of $4.3 \mathrm{~mm}$ giving a repetition rate of around $9.4 \mathrm{GHz}$ (the refractive index was 3.7 ) with a $17 \%$ absorber section. By applying a negative bias voltage to the saturable absorber section, a passive mode-locking operation was achieved for a range of currents $(75-200 \mathrm{~mA})$ and voltages $(-1$ to $-3 \mathrm{~V})$. Typically, the lasers emitted pulses of some few picoseconds and an optical spectrum of up to 200 modes. The lasers were coupled to a high-frequency photodiode, and the RF linewidth of the first four harmonics was measured using a 50 $\mathrm{GHz}$ electronic spectrum analyzer and the fitting method described in [12]. The RF line shape approximated well to a Lorentzian (Fig. 1) for the first four harmonics and broadened quadratically with the harmonic number (Fig. 1, inset), demonstrating that the $\mathrm{RF}$ spectrum is dominated by the timing jitter contribution. This validates the assumptions of [8] for these lasers.

The optical linewidth of each individual laser mode was measured through heterodyne beating of the modes with a frequency stable tunable laser source with an optical linewidth of $\sim 100 \mathrm{kHz}$. The line shape observed on the electronic spectrum analyzer approximated very well to a Lorentzian as shown in Fig. 2(a) (inset).

Figure 2(a) shows the individual mode linewidths (filled circles) of the device as a function of mode number for a gain current of $120 \mathrm{~mA}$ and a reverse bias of $-2.0 \mathrm{~V}$. The corresponding optical spectrum is shown in Fig. 2(b). For convenience we have set $n_{0}$ $=0$ in these plots. The linewidth of the passively MLL

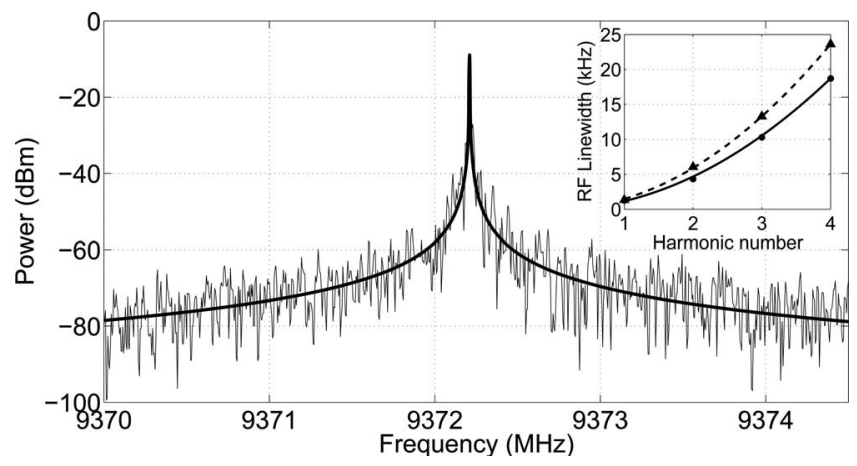

Fig. 1. RF linewidth measurement (gray curve) and the Lorentzian fit (black curve) for $150 \mathrm{~mA}$ gain current and $-1.0 \mathrm{~V}$ absorber bias. Inset, RF linewidth for the first four harmonics and predicted quadratic fit for $120 \mathrm{~mA}$ (circles, solid curve) and $160 \mathrm{~mA}$ (triangles, dotted curve) gain currents and $-1.0 \mathrm{~V}$ absorber bias. (a)

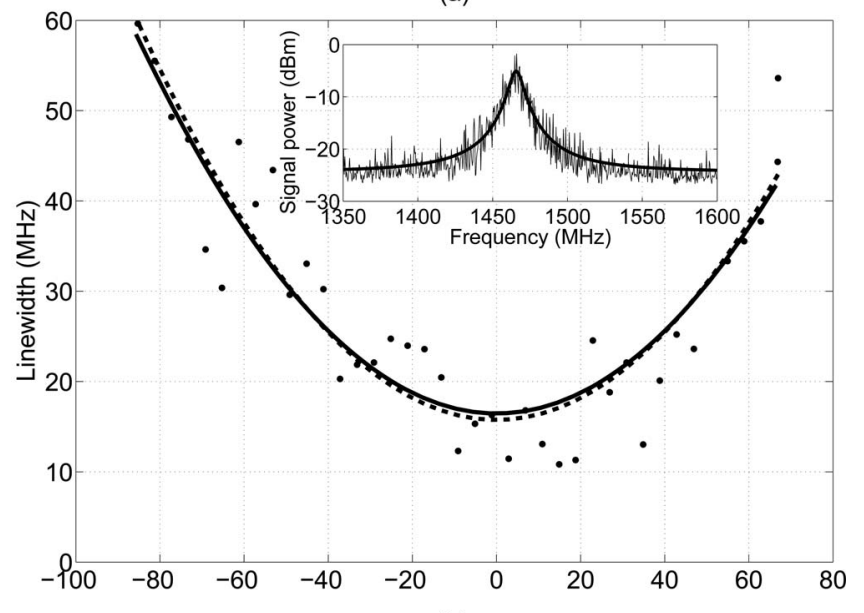

(b)

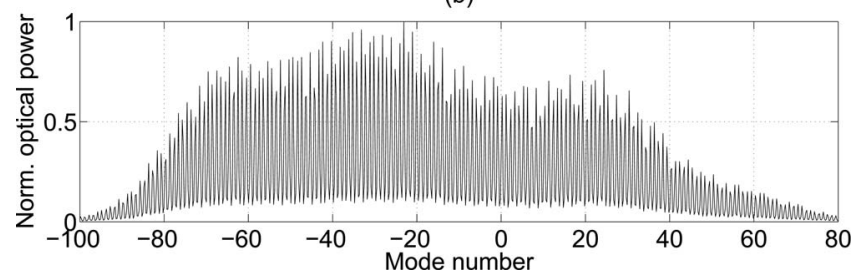

Fig. 2. (a) Individual mode linewidths of passively MLL in the vicinity of the mode number (filled circles) at a gain current of $120 \mathrm{~mA}$ and $-2.0 \mathrm{~V}$ absorber bias, parabolic fit (dotted curve), and theoretical linewidth (solid curve). (b) Corresponding optical spectrum. Inset, longitudinal-mode linewidth measurement (gray curve) and the Lorentzian fit (black curve) for $150 \mathrm{~mA}$ gain current and $-1.0 \mathrm{~V}$ absorber bias.

was of the order of tens of megahertz and demonstrated a strong parabolic dependence on the mode optical frequency. The solid curve in Fig. 2(a) shows the fitted linewidth obtained from Eq. (4) using experimental data for the RF linewidth and $\Delta \omega_{0}$ as the fitting parameter. The minimum linewidth value (for the zeroth mode) was offset from the center of the optical spectrum. This offset (which increased with the injection current) arises from temporally asymmetric chirped pulses. Briefly, from Eq. (4), the mode with the lowest linewidth is the closest to the frequency $\omega_{0}$. The center frequency of the optical spectrum is calculated from $\left|\hat{a}\left(\omega-\omega_{0}\right)\right|^{2}$. From the properties of the Fourier transform, this function is not symmetric about $\omega_{0}$ if $a(t)$ is complex (chirped pulses) and asymmetric with respect to time. Both numerical modeling [13] and frequency-resolved optical gating measurements [14] of passively mode-locked QD lasers have shown an asymmetric pulse shape and a significant pulse chirp.

Sets of individual mode linewidths were collected over a range of gain currents and bias voltages. Fits were made of the data to Eq. (4) with the RF linewidth and the minimum optical linewidth as the fitting parameters. The comparison between the directly measured and the fitted $\Delta \omega_{\mathrm{RF}, 1}$ is made in Fig. 3 where, within the accuracy of the experiment, the measured and the calculated $\mathrm{RF}$ linewidth values largely agree.

The second fitted parameter $\Delta \omega_{0}$ is plotted in a logarithmic scale in Fig. 4 for the range of currents 


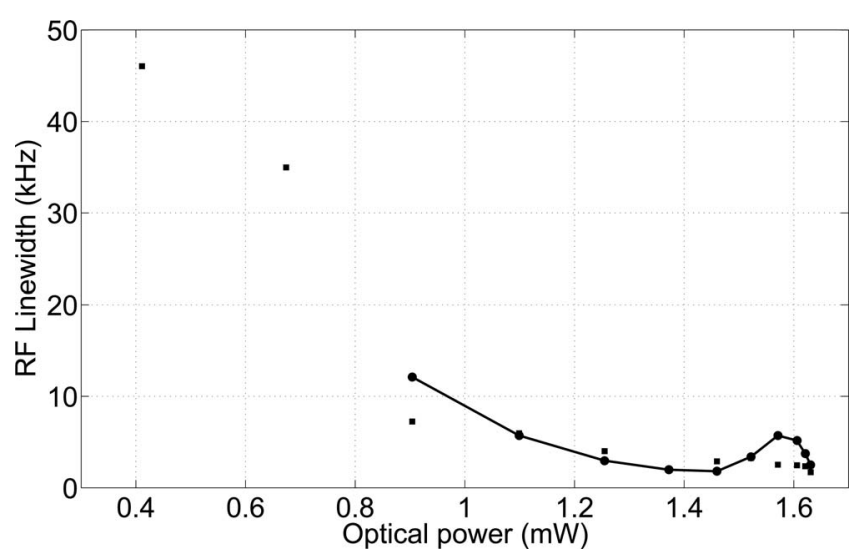

Fig. 3. Fitted (squares) and measured (circles, solid curve) $\mathrm{RF}$ linewidths for $-2.0 \mathrm{~V}$ absorber bias.

$(90-200 \mathrm{~mA})$ and absorber biases of -1.0 and $-2.0 \mathrm{~V}$. Since the threshold current was different for the different absorber biases, the results are presented as a function of the total optical power. The minimum linewidth value for the laser decreased rapidly near the threshold with increasing current, demonstrating a Schawlow-Townes-like dependence, but with a rebroadening at higher currents (higher than $1.5 I_{\mathrm{th}}$ ).

In summary, we have measured the modal optical linewidth and the RF spectrum of a QD passively MLL. We have shown the parabolic form of the optical linewidth spectrum and verified for the first time (to our knowledge) the relationship between the parabolic linewidth spectrum and the RF linewidth. This technique could be of great value in measuring the timing jitter of high repetition rate MLLs whose

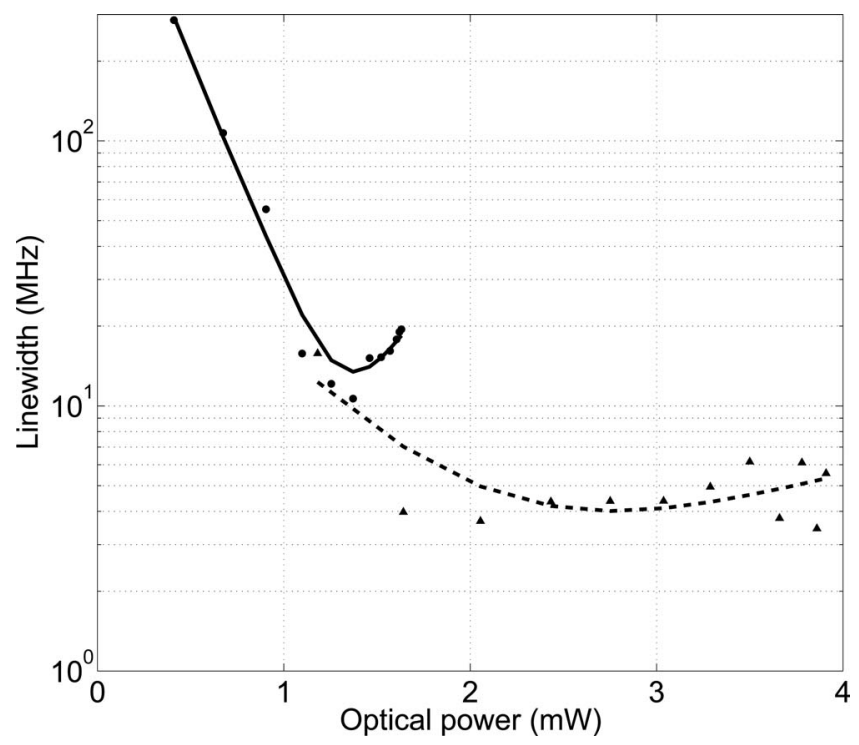

Fig. 4. Minimum value of optical linewidth for $-1.0 \mathrm{~V}$ (diamonds, dotted curve) and $-2.0 \mathrm{~V}$ (circles, solid curve) absorber biases versus total optical power. frequency exceeds that of available microwave spectrum analyzers. The deduction of the timing jitter from mode linewidth measurements can be extended to lasers with other noise mechanisms, e.g., HausGordon noise, as long as the noise source is known [15]. Phase noise dominates amplitude noise in passively mode-locked QD lasers, and the minimum modal linewidth follows a Schawlow-Townes form with a rebroadening at higher powers. The offset of the minimum linewidth mode from the center of the optical spectrum is consistent with recent reports of chirped temporally asymmetric pulses.

This work was conducted under the framework of the INSPIRE program, funded by the Irish Government's Programme for Research in Third Level Institutions, Cycle 4, National Development Plan 20072013 , and the authors also gratefully acknowledge the support of Science Foundation Ireland under contract 07/IN.1/I929.

\section{References}

1. J. Ye and S. T. Cundiff, Femtosecond Optical Frequency Comb: Principle, Operation, and Applications (Springer, 2004).

2. P. J. Delfyett, S. Gee, M. Choi, H. Izadpanah, W. Lee, S. Ozharar, F. Quinlan, and T. Yilmaz, J. Lightwave Technol. 24, 2701 (2006).

3. X. D. Huang, A. Stintz, H. Li, L. F. Lester, J. Cheng, and K. J. Malloy, Appl. Phys. Lett. 78, 2825 (2001).

4. M. Kuntz, G. Fiol, M. Lammlin, D. Bimberg, M. G. Thompson, K. T. Tan, C. Marinelli, R. V. Penty, I. H. White, V. M. Ustinov, A. E. Zhukov, Y. M. Shernyakov, and A. R. Kovsh, Appl. Phys. Lett. 85, 843 (2004).

5. E. U. Rafailov, M. A. Cataluna, and W. Sibbett, Nat. Photonics 1, 395 (2007).

6. J. K. Wahlstrand, J. T. Willits, C. R. Menyuk, and S. T. Cundiff, Opt. Express 16, 18624 (2008).

7. H. A. Haus and A. Mecozzi, IEEE J. Quantum Electron. 29, 983 (1993).

8. F. X. Kartner, U. Morgner, T. Schibli, R. Ell, H. A. Haus, J. G. Fujimoto, and E. P. Ippen, Top. Appl. Phys. 95, 73 (2004).

9. Y. Takushima, H. Sotobayashi, M. E. Grein, E. P. Ippen, and H. A. Haus, Proc. SPIE 5595, 213 (2004).

10. H. Su, L. Zhang, R. Wang, T. C. Newell, A. L. Gray, and L. F. Lester, IEEE Photon. Technol. Lett. 16, 2206 (2004).

11. M. T. Todaro, J. P. Tourrenc, S. P. Hegarty, C. Kelleher, B. Corbett, G. Huyet, and J. G. McInerney, Opt. Lett. 31, 3107 (2006).

12. F. Kéfélian, S. O’Donoghue, M. T. Todaro, J. G. McInerney, and G. Huyet, IEEE Photon. Technol. Lett. 20, 1405 (2008).

13. A. G. Vladimirov and D. Turaev, Phys. Rev. A 72, 033808 (2005).

14. Y.-C. Xin, D. J. Kane, and L. F. Lester, Electron. Lett. 44, 1255 (2008).

15. M. Ablowitz, B. Ilan, and S. T. Cundiff, Opt. Lett. 31, 1875 (2006). 